\title{
Collective cell migration: general themes and new paradigms Caren Norden ${ }^{1}$ and Virginie Lecaudey ${ }^{2}$
}

\begin{abstract}
Collective cell migration plays essential roles in embryogenesis and also contributes to disease states. Recent years have seen immense progress in understanding mechanisms and overarching concepts of collective cell migration.

Self-organization of moving groups emerges as an important common feature. This includes self-generating gradients, internal chemotaxis or mechanotaxis and contact-dependent polarization within migrating cell groups. Here, we will discuss these concepts and their applications to classical models of collective cell migration. Further, we discuss new models and paradigms of collective cell migration and elaborate on open questions and future challenges. Answering these questions will help to expand our appreciation of this exciting theme in developmental cell biology and contribute to the understanding of disease states.
\end{abstract}

\section{Addresses \\ ${ }^{1}$ Max Planck Institute of Molecular Cell Biology and Genetics, Pfotenhauerstraße 108, 01307 Dresden, Germany \\ ${ }^{2}$ Department of Developmental Biology of Vertebrates, Institute for Cell Biology and Neuroscience, Goethe University Frankfurt am Main, Frankfurt am Main, Germany \\ Corresponding authors: Norden, Caren (norden@mpi-cbg.de), Lecaudey, Virginie (lecaudey@bio.uni-frankfurt.de)}

\section{Current Opinion in Genetics \& Development 2019, 57:54-60}

This review comes from a themed issue on Dev. mechanisms, patterning and evolution

Edited by Gaspar Jekely and Maria Arnone

https://doi.org/10.1016/j.gde.2019.06.013

0959-437X/@ 2019 Elsevier Ltd. All rights reserved.

\section{Introduction}

Collective cell migration (CCM) is a fascinating process during which multiple cells move in a coordinated manner influenced by their neighbours and at the same time reacting to environmental cues. CGM occurs in many different contexts: In vitro CGM is observed in culture of epithelial monolayers [1] and 3D mammary glands [2].

In vivo, CCM is a prominent feature in many contexts of embryo development. It can also be a hallmark of disease states for example during wound healing and cancer cell invasion (reviewed in Ref. [3]). CGM in development features cells that move as groups of different sizes, shapes and adhesive properties depending on tissue context. What all CCM phenomena have in common however is that cells usually migrate over long distances. This raises the questions of how guiding cues can provide information over such long migratory paths.

Cells migrating collectively in vivo have adapted to these challenges by developing self-organizing strategies that make their migration to some extent independent of environmental cues. The last decade of CCM research has seen quite some headway in understanding these strategies and thereby the mechanisms controlling CGM during development. These were mostly based on studies of classical CCM models such as the posterior lateral line primordium ( $\mathrm{pLLP}$ ) in zebrafish, trachea development and border cell migration in Drosophila as well as blood vessel sprouting and neural crest migration in different vertebrates [4]. The mechanisms regulating CCM have been discussed in a number of recent reviews [4-9]. Instead, in this review we will focus on general mechanisms driving CGM that have been identified in recent studies of diverse model systems. We will emphasize self-generated and self-propagating processes that emerge as common themes in many CGM models.

In addition to classical models of CCM, we also discuss the lately discovered rim migration during optic cup morphogenesis, a particular CCM phenomenon. We close by eluding to yet unresolved questions in our understanding of CCM and suggest approaches to address these in future studies.

\section{Common themes of CCM}

\section{Migration guided by self-generated gradients}

Like single cells, groups of cells often move in the direction determined by a concentration gradient of a guidance molecule. Such gradient can result from the regulated expression of the guidance molecule along the path, or from its diffusion from a producing source to a degrading sink. The sink is a group of cells that removes the guidance molecule either by endocytosis, enzymatic degradation or flow. In a number of cases, the migrating cells themselves degrade the chemoattractant and this way generate their own gradient from an initially non-graded source of a guidance molecule (Figure 1a-i)

Slugs of the slime mould Dictyostelium are comprised of thousands of migrating cells that move as a group in 


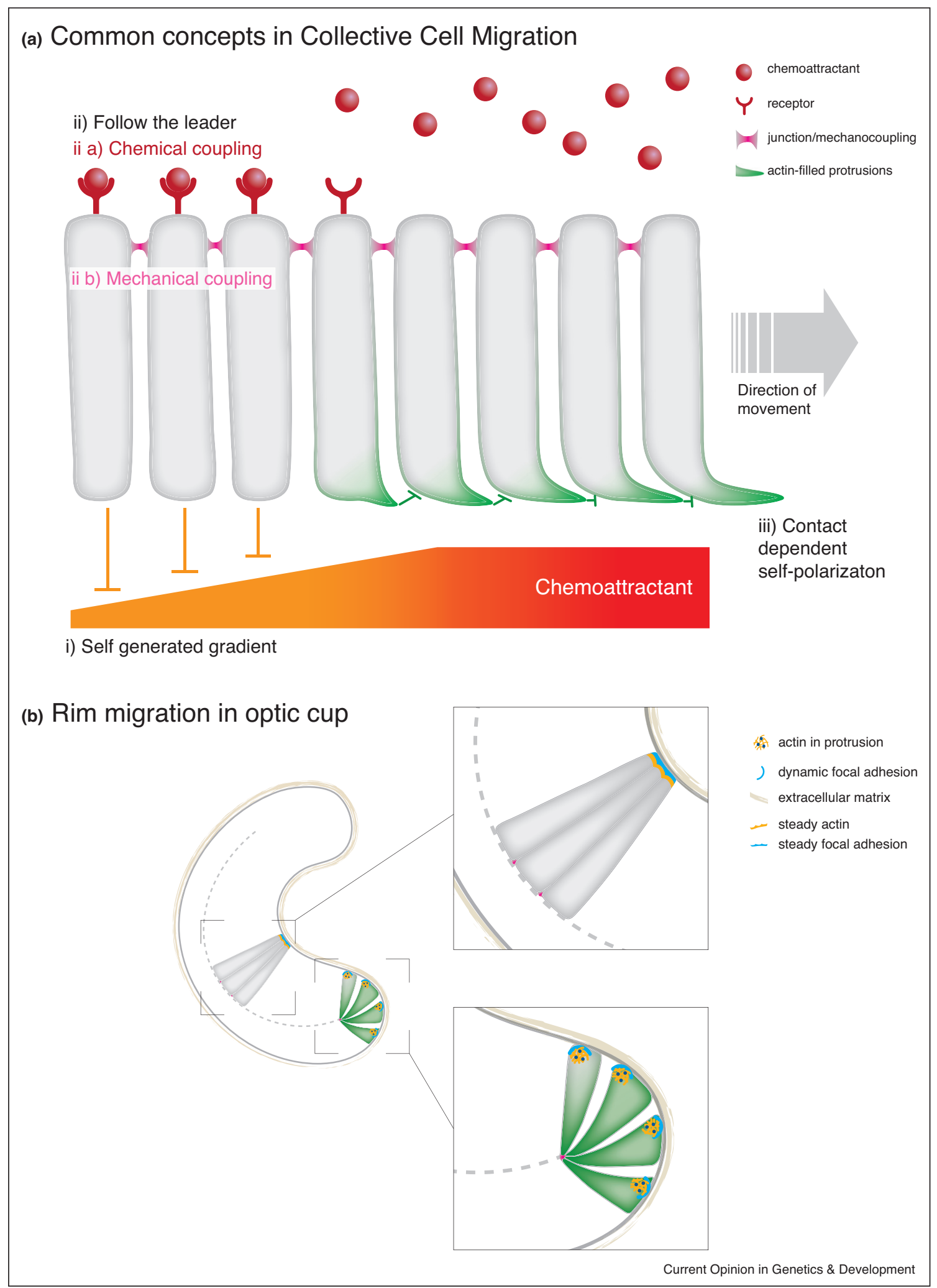

(a) Schematic summarizing the common concepts in collective cell migration described in the review. Depending on the CCM model, they occur alone or in different combinations: i) When the migrating cells (or a subset thereof) degrade the guidance cues, a self-generated gradient forms and moves along with the migrating cell group. ii) The 'Follow-the-leader' mode refers to cases, in which follower cells are either chemically (a) or 
response to a cAMP gradient. Dictyostelium cells need to secrete a phosphodiesterase to degrade cAMP suggesting they generate a cAMP gradient [10]. In addition, Dictyostelium cells chemotactically respond to folate and at the same time express a folate deaminase that degrades it, thus generating a folate gradient. These experiments combined with computational simulations have shown that Dictyostelium slugs generate their own steep, local gradient $\left[11^{\bullet \bullet}\right]$.

Similarly, cells of the pLLP of zebrafish are not guided by a pre-existing gradient. They respond to the chemokine Cxcl12a, which is initially evenly distributed [12]. The required gradient is established by the cells in the trailing part of the pLLP, which express the G-protein-coupled receptor Cxcr7b. Here Cxcr7b functions as a decoy receptor, that is, it efficiently binds and removes Cxcl12a from the extracellular space without triggering an intracellular signalling cascade, thus acting as an internal sink $[13,14]$. This generates a Cxcl12a gradient to which the pLLP cells respond via classical chemotactic response using the other G-protein-coupled receptor Cxcr $4 b\left[15^{\bullet}, 16^{\circ}\right]$. Self-generated gradients have two major advantages: they are localized and steep, and they move along with the migrating cells. In both, Dictyostelium and the pLLP, these self-generated gradients promote robust CGM over long distances.

Self-generated gradients are also involved in CCM in pathological contexts. Malignant melanoma cells, for example, which cause the most aggressive form of skin cancer, degrade their own chemoattractant, the phospholipid signalling molecule lysophosphatidic acid [17 $\left.{ }^{\bullet \bullet}\right]$. Here, the chemoattractant is not sequestered by a decoy receptor but enzymatically degraded by the lipid phosphatase LPP3 [18 $]$.

Further interesting characteristics of self-generated gradient are that: (i) 'more is not better' since higher chemoattractant concentration leads to longer times for cells to form a gradient to which they can respond $\left[18^{\circ}\right]$ and (ii) a critical mass of cells needs to be present to remove the chemoattractant and thus to initiate migration.

\section{Follow the leader!}

Beside self-generated gradients, collectively migrating cells often produce a relay signal to coordinate the movement of the front cells with the movement of cells in the back. In this mode, referred to as 'followthe-leader', the front cells respond directly to the chemoattractant and either produce a secondary chemoattractant or mechanically pull on the trailing cells inducing them to follow (Figure 1a-ii, a and b).

Dictyostelium slug migration is largely based on such a relay mechanism. While leader cells respond to a cAMP gradient, they also produce cAMP. Thus, cells at the tip of the slug form an internal source of the chemoattractant that travels in waves through the moving group. Coordination of cell movement is achieved by the fact that non-front cells follow this internal gradient [19].

A similar mechanism has been characterized in zebrafish pLLP. While all cells expressing the Cxcr4b receptor can respond to the self-generated gradient of Cxcl12a, transplant experiments have shown that as little as two Cxcr4b-expressing cells at the leading front are sufficient to rescue migration of the entire pLLP [12]. This suggests that non-front cells respond to a relay signal produced by the leading cells. This signal was proposed to be the FGF ligands expressed by the leading cells [20]. Overall, this mechanism makes the pLLP a very interesting selforganizing system in which the leading cells steer the group in response to a chemokine gradient generated by the trailing cells, while in turn the trailing cells follow the FGF-expressing leading cells (Figure 1a, ii a).

As mentioned above, in other examples of collective cell migration, leaders and followers are mechanically coupled, for example by cadherin-based cell-cell junctions (Figure 1a, ii b). During Drosophila trachea formation and vertebrate angiogenesis for example, leaders (also referred to as tip cells) are initially selected based on their response to an external source of an FGF or VEGF chemoattractant [21,22]. This fate is maintained by Notch-mediated lateral inhibition, which prevents other cells to react to the chemoattractant [23-25]. In response to the chemoattractant, tip cells form large protrusions that drive CCM. Follower cells that are mechanically coupled to the tip cells [26-29] contribute to the elongation of the branch by intercalation (trachea) or proliferation (blood vessels) [21,30-32]. In Drosophila trachea migration, the tip cell generates sufficient traction forces to drive intercalation of the mechanically coupled stalk

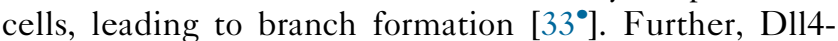
Notch signalling plays an important role for leader cell selection in epithelial monolayers [34].

Within the border cell cluster of the Drosophila egg chamber, cells are similarly mechanically coupled via E-cadherin-mediated cell-cell junctions. The border cell cluster is composed of $6-8$ actively migrating border cells

\footnotetext{
(Figure 1 Legend Continued) mechanically (b) coupled to the leaders and iii) Contact-dependent polarization inhibits the formation of protrusions at cell-cell contacts and promotes it at contact-free locations, thus ensuring coordination of migration within the cell group.

(b) Schematic of rim cell migration during optic cup formation. Cells move from the outer side of the optic vesicle into the neuroepithelium using a specialized form of collective cell migration. This collective cell migration depends on dynamic focal adhesions (blue), actin rich protrusions (orange) and contact to the underlying extracellular matrix (grey).
} 
that surround two non-motile polar cells and move up a gradient of EGF/PVF1. E-cadherin-mediated mechanical coupling between border cells transmits directional information from the leading cell to the other border cells $\left[35^{\bullet \bullet}\right]$.

How do cell-cell contacts transmit directional information from one cell to another for coordinated movement of several cells? Recent work shows that the tension exerted by a migrating cell on cells that are mechanically coupled to it plays an important role. In cultured epithelial cells, the tumour suppressor protein Merlin re-localizes from cell-cell junctions to the cytoplasm at the onset of migration. This re-localization is triggered by the pulling forces exerted by the front cell and acting on its neighbours. This pulling force depends on actomyosin contractility which triggers polarized Rac1 activation and lamellipodium formation in the back cell. This back cell consequently starts to migrate and in turn pulls on the next cell, further transmitting the directional information [36 $]$.

Thus, 'follow-the-leader' relay signals, whether chemical or biomechanical, underlie the propagation of directional information within the migrating cell group, thereby ensuring coordinated movement.

\section{Contact-dependent self-polarization}

While junctions between border cells transmits directionality information (see above), E-cadherin-mediated contacts between border cells and polar cells are important to provide polarity to each border cells by biasing their protrusions in a radial outward direction [35 $\left.{ }^{\circ}\right]$. This contact-dependent cell polarization is another important mechanism that enables epithelial cells to coordinate their movements in an autonomous manner during CGM (Figure 1a, iii). Contact-dependent cell polarization ensures that the formation of protrusions is inhibited at cell-cell contacts and promoted at contact free locations. By itself, this mechanism would lead to a radial polarization of an epithelial cluster and therefore needs to be combined with other mechanisms providing directionality to the group. In border cells, contact-dependent cell polarization does not only occur between border cells and polar cells $\left[35^{\bullet \bullet}\right.$ ] but also at border cell — border cell contacts at which protrusion formation is actively suppressed by the Hippo pathway controlling actin polarization [37]. In the Drosophila trachea, protrusions also orient in the direction of migration, away from cell-cell contacts [38]. In the zebrafish pLLP, isolating the leading region of the migrating group using laser ablation leads to stalling of the isolated region and the formation of protrusions at the newly generated free edges. When the back of the pLLP reinitiates migration and collides with the stalled leading region, these ectopic protrusions are inhibited, and polarity and migration of the leading region is restored [20]. Together, this strongly suggests a contact-dependent polarization of the pLLP cells.
Remarkably, a very similar behaviour was observed for prechordal plate cells migrating towards the animal pole during zebrafish gastrulation. Prechordal plate cells transplanted as small groups ahead of the endogenous plate form active, but non-directed protrusions. However, as soon as they are in contact with the endogenous plate, cells re-orient towards the animal pole and migrate at the front of the plate, very similar to the isolated leading region of the pLLP [39]. These cells thus require an instructive directional signal that is provided by contact to the trailing cells. A contact-dependent cell polarization mechanism has also been identified in Xenopus mesendodermal cell migration. Applying force on isolated mesendodermal cells in vitro using C-cadherin-coated beads induces cell protrusions and migration in the opposite direction [40]. This in turn creates a positive mechanical feedback loop that could account for the transmission of polarity information within a group of cells in a fully autonomous manner.

It should be noted that contact-dependent cell polarization shares many similarities with contact-inhibition of locomotion, a mechanism that plays an essential role in the CCM of mesenchymal cells such as neural crest cells $[7,9,41,42]$. This underlined the idea that indeed strategies ensuring successful CCM are widely conserved.

\section{Emerging models: rim migration in the optic cup}

The previous chapters described common themes within classical CCM models and phenomena. Excitingly however, new CCM phenomena are still being discovered in different tissue contexts that enable enhancing the picture of this interesting process. One prominent example is rim migration, a crucial feature of zebrafish optic cup formation $\left[43^{\bullet \bullet}\right.$. Zebrafish optic cup formation involves the restructuring of the pouch-like optic vesicle consisting of two opposing neuroepithelia into the hemispheric optic cup. During optic cup formation some cells change their position from one side of the pouch to the other in the so-called rim involution thereby becoming part of the retinal neuroepithelium. This phenomenon was first discovered in 2009 in zebrafish [44] and was later shown to also occur during chick optic cup formation [45]. However, it was unclear whether it was driven purely by epithelial flow [46] or contained active collective migration components. A recent study clarified that rim involution is actually a form of CCM as cells stay apically attached while they at the same time use basal dynamic membrane protrusions to move directionally [43 $\left.{ }^{\bullet \bullet}\right]$ (Figure 1b). When these protrusions or the underlying extracellular matrix were perturbed, rim migration was severely hampered leading to serious problems in optic cup formation. It is possible, that dependence on cellmatrix interplay is also at play in other CCM phenomena; however, this has not yet been extensively explored (see below). Interestingly, as opposed to many other CCM 
phenomena (see above) the optic vesicle is a closed continuous epithelial sheet and thus lacks a specific leading edge or leader cells (Figure 1b). This raises the question how the migratory process is nevertheless limited to the rim zone and how directionality of cell movements is assured. As currently nothing is known about the chemical or mechanical cues that drive rim migration, these will be interesting avenues to explore in future studies. Here it will help to build on the knowledge and examples summarized above when planning experimental strategies. The finding that rim migration is a specialised form of CCM also indicates that there are more examples of CCM driving developmental and morphogenetic processes yet to be discovered.

\section{Challenges and open questions}

The last decade has seen further improvement in our comprehension of CCM and the establishment of common themes and differences in this process.

One current caveat is however that especially in vivo studies so far mainly took an 'apical-centred' approach. For the convenience of imaging, cells are often followed from their apical surface. This is for example true for studies of pLLP in zebrafish [47] or border cell migration in Drosophila [48]. However, especially to fully understand the cell biological aspects of epithelial CCM, it would be important to also visualize basal cell dynamics. Many epithelial cells undergoing CCM are moving along a basal extracellular matrix and the example of rim migration during optic cup formation clearly shows the dependence of CCM on basal cell protrusions and the maintenance of the extracellular matrix (Figure 1b). Thus, the cell-matrix interplay needs to be explored. To achieve this, two prerequisites have to be fulfilled: 1) image acquisition needs to also include basolateral and basal views. This might be challenging in some model systems, but with ever improving imaging solutions and recent advances in light sheet microscopy [49,50] will become more feasible, 2) to understand cellular dynamics and interplay with the extracellular matrix, in vivo markers of the latter are needed. While some extracellular matrix lines have been used in Caenorhabditis elegans and Drosophila [51,52], especially vertebrate systems would profit from the generation of more extracellular matrix live-imaging tools.

Finally, as outlined above, understanding the chemical cues that drive CCM has been the focus of many previous studies. In contrast, the mechanical and physical cues that drive CCM are less explored. However, the field of mechanobiology has been constantly growing in recent years [8] and techniques like laser ablation [53,54] and mechanosensors as readout for overall tissue stresses and mechanical cues $[55,56]$ become more user-friendly. Thus, deciphering the mechanisms establishing, maintaining and propagating directionality during CGM in different tissue and organismal contexts in addition to or instead of chemical cues will become easier in the near future.

Taken together, while progress has been made, lots of open questions remain, meaning that CGM will continue to be an exciting field of developmental cell biology research for years to come.

\section{Conflict of interest statement}

Nothing declared.

\section{Acknowledgements}

We thank Karen Soans and Franziska Friedrich from the MPI-CBG MTO department for illustrations.

C.N. was supported by the MPI-CBG and the German Research Foundation (NO 1068/3-1) and under Germanýs Excellence Strategy EXC-2068 390729961- Cluster of Excellence Physics of Life of TU Dresden.

\section{References and recommended reading}

Papers of particular interest, published within the period of review, have been highlighted as:

- of special interest

$\bullet$ of outstanding interest

1. Farooqui R, Fenteany G: Multiple rows of cells behind an epithelial wound edge extend cryptic lamellipodia to collectively drive cell-sheet movement. J Cell Sci 2005, 118:5163

2. Ewald AJ, Brenot A, Duong M, Chan BS, Werb Z: Collective epithelial migration and cell rearrangements drive mammary branching morphogenesis. Dev Cell 2008, 14:570-581.

3. Friedl P, Gilmour DT: Collective cell migration in morphogenesis, regeneration and cancer. Nat Rev Mol Cell Biol 2009, 10:445-457.

4. Scarpa E, Mayor R: Collective cell migration in development. $J$ Cell Biol 2016, 212:143-155.

5. Olson HM, Nechiporuk AV: Using zebrafish to study collective cell migration in development and disease. Front Cell Dev Biol 2018, 6:83.

6. Theveneau E, Linker C: Leaders in collective migration: are front cells really endowed with a particular set of skills? F1000Res 2017, 6:1899.

7. Theveneau E, Mayor R: Collective cell migration of epithelial and mesenchymal cells. Cell Mol Life Sci 2013, 70:3481 http://dx doi.org/10.1007/s00018-012-1251-7.

8. Ladoux B, Mège R-M, Trepat X: Front-rear polarization by mechanical cues: from single cells to tissues. Trends Cell Biol 2016, 26:420-433

9. Stramer B, Mayor R: Mechanisms and in vivo functions of contact inhibition of locomotion. Nat Rev Mol Cell Biol 2017, 18:43-55 http://dx.doi.org/10.1038/nrm.2016.118.

10. Dormann D, Weijer CJ: Chemotactic cell movement during Dictyostelium development and gastrulation. Curr Opin Genet Dev 2006, 16:367-373.

11. Tweedy L, Knecht DA, Mackay GM, Insall RH: Self-generated

- chemoattractant gradients: attractant depletion extends the range and robustness of chemotaxis. PLOS Biol 2016, 14: e1002404The authors combine experiments with Dictyostelium cells and computational simulations to analyse self-generated gradients. They show that chemoattractant degradation by migrating cells create steep local gradients that move with the migrating cells enabling robust and long-distance migration. 
12. Haas P, Gilmour DT: Chemokine signaling mediates selforganizing tissue migration in the zebrafish lateral line. Dev Cell 2006, 10:673-680.

13. Dambly-Chaudiere C, Cubedo N, Ghysen A: Control of cell migration in the development of the posterior lateral line: antagonistic interactions between the chemokine receptors CXCR4 and CXCR7/RDC1. BMC Dev Biol 2007, 7:23.

14. Valentin G, Haas P, Gilmour DT: The chemokine SDF1a coordinates tissue migration through the spatially restricted activation of Cxcr7 and Cxcr4b. Curr Biol 2007, 17:1026-1031.

15. Donà $E$, Barry JD, Valentin G, Quirin $C$, Khmelinskii A, Kunze A,

- Durdu S, Newton LR, Fernández-Miñán A, Huber W et al.: Directional tissue migration through a self-generated chemokine gradient. Nature 2013 http://dx.doi.org/10.1038/ nature12635The authors apply a tandem fluorescent protein timer approach to the pLLP as a CCM model to measure ligand-triggered receptor turnover in vivo. They show that cells in the back of the pLLP, which express the decoy receptor Cxcr7b, function as a sink and generate a local and robust gradient of the $\mathrm{Cxcl12a}$ chemokine across the pLLP. The pLLP cells which express the other GPCR Cxcr4b respond to it to this self-generated gradient and establish a selfdirected and long-lasting migration.

16. Venkiteswaran G, Lewellis SW, Wang J, Reynolds E, Nicholson C,

-. Knaut H: Generation and dynamics of an endogenous, selfgenerated signaling gradient across a migrating tissue. Cell $2013,155: 674-687$ The authors drew very similar conclusions as in Ref. [15] using the internalization of a fluorescent Cxcr4b fusion protein as a Cxcl12a in vivo sensor in the pLLP system.

17. Muinonen-Martin AJ, Susanto O, Zhang Q, Smethurst E, Faller WJ

-• Veltman DM, Kalna G, Lindsay C, Bennett DC, Sansom OJ et al.: Melanoma cells break down LPA to establish local gradients that drive chemotactic dispersal. PLOS Biol 2014, 12: e1001966The authors use a chamber-based assay and find that melanoma cells migrate efficiently away from one another by positive chemotaxis. They show that melanoma cells rapidly break down lysophosphatidic acid in their environment and act as a sink to generate their own outward gradients. This self-directed migration is in agreement with the observation that metastasis of melanoma largely depends on the thickness of the tumour.

18. Susanto O, Koh YWH, Morrice N, Tumanov S, Thomason PA,

- Nielson M, Tweedy L, Muinonen-Martin AJ, Kamphorst JJ, Mackay GM et al.: LPP3 mediates self-generation of chemotactic LPA gradients by melanoma cells. J Cell Sci 2017 130:3455-3466Here the authors identify the lipid phosphatase LPP3 as the key enzyme enabling melanoma cells to break down lysophosphatidic acid inside the tumor and thus generate their own lysophosphatidic acid gradient promoting their outward chemotactic migration.

19. Dormann D, Weijer CJ: Propagating chemoattractant waves coordinate periodic cell movement in Dictyostelium slugs. Development 2001, 128:4535-4543.

20. Dalle Nogare D, Somers K, Rao S, Matsuda M, Reichman-Fried M, Raz E, Chitnis AB: Leading and trailing cells cooperate in collective migration of the zebrafish posterior lateral line primordium. Development 2014, 141:3188-3196.

21. Gerhardt $H$, Golding $M$, Fruttiger M, Ruhrberg C, Lundkvist A, Abramsson A, Jeltsch M, Mitchell C, Alitalo K, Shima D et al: VEGF guides angiogenic sprouting utilizing endothelial tip cell filopodia. J Cell Biol 2003, 161:1163-1177.

22. Ribeiro C, Ebner A, Affolter M: In vivo imaging reveals different cellular functions for FGF and Dpp signaling in tracheal branching morphogenesis. Dev Cell 2002, 2:677-683.

23. Ghabrial AS, Krasnow MA: Social interactions among epithelial cells during tracheal branching morphogenesis. Nature 2006, 441:746-749.

24. Hellström M, Phng L-K, Hofmann JJ, Wallgard E, Coultas L, Lindblom P, Alva J, Nilsson A-K, Karlsson L, Gaiano N et al.: DII4 signalling through Notch1 regulates formation of tip cells during angiogenesis. Nature 2007, 445:776-780.

25. Jakobsson L, Franco CA, Bentley K, Collins RT, Ponsioen B, Aspalter IM, Rosewell I, Busse M, Thurston G, Medvinsky A et al.:
Endothelial cells dynamically compete for the tip cell position during angiogenic sprouting. Nat Cell Biol 2010, 12:943-953.

26. Bentley K, Franco CA, Philippides A, Blanco R, Dierkes M, Gebala V, Stanchi F, Jones M, Aspalter IM, Cagna G et al.: The role of differential VE-cadherin dynamics in cell rearrangement during angiogenesis. Nat Cell Biol 2014, 16:309-321.

27. Cao J, Ehling M, März S, Seebach J, Tarbashevich K, Sixta T, Pitulescu ME, Werner A-C, Flach B, Montanez E et al.: Polarized actin and VE-cadherin dynamics regulate junctional remodelling and cell migration during sprouting angiogenesis. Nat Commun 2017, 8:2210.

28. Tanaka-Matakatsu M, Uemura T, Oda H, Takeichi M, Hayashi S: Cadherin-mediated cell adhesion and cell motility in Drosophila trachea regulated by the transcription factor Escargot. Development 1996, 122:3697-3705.

29. Uemura T, Oda H, Kraut R, Hayashi S, Kotaoka Y, Takeichi M: Zygotic Drosophila E-cadherin expression is required for processes of dynamic epithelial cell rearrangement in the Drosophila embryo. Genes Dev 1996, 10:659-671.

30. Caussinus E, Colombelli J, Affolter M: Tip-cell migration controls stalk-cell intercalation during Drosophila tracheal tube elongation. Curr Biol 2008, 18:1727-1734.

31. Costa G, Harrington KI, Lovegrove HE, Page DJ, Chakravartula S, Bentley K, Herbert SP: Asymmetric division coordinates collective cell migration in angiogenesis. Nat Cell Biol 2016, 18:1292-1301.

32. Affolter M, Zeller R, Caussinus E: Tissue remodelling through branching morphogenesis. Nat Rev Mol Cell Biol 2009, 10:831 842.

33. Ochoa-Espinosa A, Harmansa S, Caussinus E, Affolter M: Myosin - II is not required for Drosophila tracheal branch elongation and cell intercalation. Development 2017, 144:2961-2968Using a nanobody-based approach to knock-down non-muscle myosin II specifically in Drosophila tracheal cells, the authors show that dorsal branch elongation occurs independently of non-muscle myosin IIdependent forces demonstrating that tip cell migration produces the necessary forces for stalk cell intercalation and branching.

34. Riahi R, Sun J, Wang S, Long M, Zhang DD, Wong PK: Notch1DII4 signalling and mechanical force regulate leader cell formation during collective cell migration. Nat Commun 2015, 6:6556.

35. Cai D, Chen S-C, Prasad M, He L, Wang X, Choesmel-

-• Cadamuro V, Sawyer JK, Danuser G, Montell DJ: Mechanical feedback through E-cadherin promotes direction sensing during collective cell migration. Cell 2014, 157:1146-1159Here the authors want to determine the role of E-cadherin in collective migration of cells between other cells. For this, they use the Drosophila border cells which migrate as a small cluster surrounding two polar cells and using nurse cells) as a substrate. They show that E-cadherin plays a crucial role in border cell chemotaxis at different levels: between border cells to transmit directional information from leader to followers, between border cells and nurse cells to stabilize directionally persistent movement and between border cells and polar cells to hold the cluster together and polarize individual border cells outward.

36. Das T, Safferling K, Rausch S, Grabe N, Boehm H, Spatz JP: A - molecular mechanotransduction pathway regulates collective migration of epithelial cells. Nat Cell Biol 2015, 17:276-287Here the authors show that the tumour suppressor protein Merlin coordinates the migration of multiple cells by acting as a mechanochemical transducer. They show that the pulling forces exerted by a migrating cell on the cells mechanically coupled to it triggers the translocation of Merlin from the cell cortex to the cytoplasm. This leads to Rac polarization and lamellipodium formation and spreads to the multicellular length scale.

37. Lucas EP, Khanal I, Gaspar P, Fletcher GC, Polesello C, Tapon N, Thompson BJ: The Hippo pathway polarizes the actin cytoskeleton during collective migration of Drosophila border cells. J Cell Biol 2013, 201:875-885.

38. Lebreton G, Casanova J: Specification of leading and trailing cell features during collective migration in the Drosophila trachea. J Cell Sci 2014, 127:465-474. 
39. Dumortier JG, Martin S, Meyer D, Rosa FM, David NB: Collective mesendoderm migration relies on an intrinsic directionality signal transmitted through cell contacts. Proc Natl Acad Sci U S A 2012, 109:16945-16950 http://dx.doi.org/10.1073/ pnas.1205870109.

40. Weber GF, Bjerke MA, Desimone DW: A mechanoresponsive cadherin-keratin complex directs polarized protrusive behavior and collective cell migration. Dev Cell 2012, 22:104115.

41. Roycroft A, Mayor R: Molecular basis of contact inhibition of locomotion. Cell Mol Life Sci 2016, 73:1119-1130.

42. Scarpa E, Szabó A, Bibonne A, Theveneau E, Parsons M, Mayor R: Cadherin switch during EMT in neural crest cells leads to contact inhibition of locomotion via repolarization of forces. Dev Cell 2015, 34:421-434.

43. Sidhaye J, Norden C: Concerted action of neuroepithelial basal -. shrinkage and active epithelial migration ensures efficient optic cup morphogenesis. eLife 2017, 6The authors for the first time unambiguously show that a) rim migration is crucial for overall optic cup formation and $b$ ) that it is an example of active collective cel migration. By combining elegant in vivo imaging with genetic and pharmacological manipulations they dissect different aspects of rim migration giving insights into the cell biology of this process.

44. Picker A, Cavodeassi F, Machate A, Bernauer S, Hans S, Abe G, Kawakami K, Wilson SW, Brand M: Dynamic coupling of pattern formation and morphogenesis in the developing vertebrate retina. PLoS Biol 2009, 7:e1000214.

45. Kwan KM, Otsuna H, Kidokoro H, Carney KR, Saijoh Y, Chien C-B: A complex choreography of cell movements shapes the vertebrate eye. Development 2012, 139:359-372

46. Heermann S, Schütz L, Lemke S, Krieglstein K, Wittbrodt J: Eye morphogenesis driven by epithelial flow into the optic cup facilitated by modulation of bone morphogenetic protein. eLife 2015, 4.
47. Lecaudey V, Cakan-Akdogan G, Norton WHJ, Gilmour DT: Dynamic Fgf signaling couples morphogenesis and migration in the zebrafish lateral line primordium. Development 2008 135:2695-2705.

48. Prasad M, Wang X, He L, Montell DJ: Border cell migration: a model system for live imaging and genetic analysis of collective cell movement. Methods Mol Biol 2011, 769:277-286.

49. Power RM, Huisken J: A guide to light-sheet fluorescence microscopy for multiscale imaging. Nat Methods 2017, 14:360373.

50. Follain G, Mercier L, Osmani N, Harlepp S, Goetz JG: Seeing is believing - multi-scale spatio-temporal imaging towards in vivo cell biology. J Cell Sci 2017, 130:23-38.

51. Matsubayashi Y, Louani A, Dragu A, Sánchez-Sánchez BJ, SernaMorales E, Yolland L, Gyoergy A, Vizcay G, Fleck RA, Heddleston JM et al.: A moving source of matrix components is essential for de novo basement membrane formation. Curr Biol 2017, 27:3526-3534.e4.

52. Clay MR, Sherwood DR: Basement membranes in the worm: a dynamic scaffolding that instructs cellular behaviors and shapes tissues. Curr Top Membr 2015, 76:337-371.

53. Shivakumar PC, Lenne P-F: Laser ablation to probe the epithelial mechanics in Drosophila. Methods Mol Biol 2016, 1478:241-251

54. Colombelli J, Solon J: Force communication in multicellular tissues addressed by laser nanosurgery. Cell Tissue Res 2013 352:133-147.

55. Cost A-L, Khalaji S, Grashoff C: Genetically encoded FRETbased tension sensors. Curr Protoc Cell Biol 2019, 83:e85 http:// dx.doi.org/10.1002/cpcb.85.

56. Freikamp A, Mehlich A, Klingner C, Grashoff C: Investigating piconewton forces in cells by FRET-based molecular force microscopy. J Struct Biol 2017, 197:37-42. 